J. Nonlinear Var. Anal. 3 (2019), No. 1, pp. 61-78

Available online at http://jnva.biemdas.com

https://doi.org/10.23952/jnva.3.2019.1.07

\title{
MEAN CONVERGENCE THEOREMS FOR NEW MAPPINGS IN A BANACH SPACE
}

\author{
TOSHIHARU KAWASAKI ${ }^{1,2}$ \\ ${ }^{1}$ College of Engineering, Nihon University, Fukushima 963-8642, Japan \\ ${ }^{2}$ Faculty of Engineering, Tamagawa University, Tokyo 194-8610, Japan \\ Dedicated to Professor Wataru Takahashi on the occasion of his 75th birthday
}

\begin{abstract}
In this paper, we introduce some extensions of mean convergence theorems without convexity. Several fixed point theorems are established in the framework of Banach spaces.
\end{abstract}

Keywords. Fixed point; Skew-acute point; Mean convergence theorem; Generalized pseudocontraction; Banach space.

2010 Mathematics Subject Classification. 47H10.

\section{INTRODUCTION}

In [21] Takahashi and Takeuchi introduced a concept of attractive points in a Hilbert space. Let $H$ be a real Hilbert space, let $C$ be a nonempty subset of $H$ and let $T$ be a mapping from $C$ into $H$. Recall that $x \in H$ is called an attractive point of $T$ if

$$
\|x-T y\| \leq\|x-y\|
$$

for any $y \in C$. Let

$$
A(T)=\{x \in H \mid\|x-T y\| \leq\|x-y\| \text { for any } y \in C\} .
$$

Moreover they proved that the Baillon type ergodic theorem [2] for generalized hybrid mappings [16] without convexity of set $C$. Recall that a mapping $T$ from $C$ into $H$ is said to be generalized hybrid if there exist $\alpha, \beta \in \mathbb{R}$ such that

$$
\alpha\|T x-T y\|^{2}+(1-\alpha)\|x-T y\|^{2} \leq \beta\|T x-y\|^{2}+(1-\beta)\|x-y\|^{2}
$$

for any $x, y \in C$. Such a mapping is said to be $(\alpha, \beta)$-generalized hybrid. The class of all generalized hybrid mappings is a new class of nonlinear mappings including nonexpansive mappings, nonspreading mappings [18] and hybrid mappings [20]. Recall that a mapping $T$ from $C$ into $H$ is said to be nonexpansive if

$$
\|T x-T y\| \leq\|x-y\|
$$

for any $x, y \in C$. It is said to be nonspreading if

$$
2\|T x-T y\|^{2} \leq\|T x-y\|^{2}+\|T y-x\|^{2}
$$

E-mail address: toshiharu.kawasaki@ nifty.ne.jp

Received April 6, 2018; Accepted February 20, 2019.

(C)2019 Journal of Nonlinear and Variational Analysis 
for any $x, y \in C$. It is said to be hybrid if

$$
3\|T x-T y\|^{2} \leq\|x-y\|^{2}+\|T x-y\|^{2}+\|T y-x\|^{2}
$$

for any $x, y \in C$. It is obvious that every nonexpansive mapping is $(1,0)$-generalized hybrid, every nonspreading mapping is $(2,1)$-generalized hybrid and every hybrid mapping is $\left(\frac{3}{2}, \frac{1}{2}\right)$-generalized hybrid.

Motivated these mappings, Kawasaki and Takahashi in [13] introduced a new class of mappings, called widely more generalized hybrid mappings, than the class of all generalized hybrid mappings. Recall that a mapping $T$ from $C$ into $H$ is widely more generalized hybrid if there exist $\alpha, \beta, \gamma, \delta, \varepsilon, \zeta, \eta \in \mathbb{R}$ such that

$$
\begin{aligned}
& \alpha\|T x-T y\|^{2}+\beta\|x-T y\|^{2}+\gamma\|T x-y\|^{2}+\delta\|x-y\|^{2} \\
& \quad+\varepsilon\|x-T x\|^{2}+\zeta\|y-T y\|^{2}+\eta\|(x-T x)-(y-T y)\|^{2} \leq 0
\end{aligned}
$$

for any $x, y \in C$. Such a mapping is said to be $(\alpha, \beta, \gamma, \delta, \varepsilon, \zeta, \eta)$-widely more generalized hybrid. This class includes the class of all generalized hybrid mappings and also the class of all $k$-pseudo-contractions [3] for $k \in[0,1]$. Recall that a mapping $T$ from $C$ into $H$ is said to be $k$-pseudocontractive if

$$
\|T x-T y\|^{2} \leq\|x-y\|^{2}+k\|(x-T x)-(y-T y)\|^{2}
$$

for any $x, y \in C$. Every $(\alpha, \beta)$-generalized hybrid mapping is $(\alpha, 1-\alpha,-\beta, \beta-1,0,0,0)$-widely more generalized hybrid. Every $k$-pseudo-contraction is $(1,0,0,-1,0,0,-k)$-widely more generalized hybrid. Moreover they proved some fixed point theorems (see [5, 6, 7, 8, 9, 10, 12, 13, 14, 15] and some ergodic theorems (see $[5,6,12,13,14]$ ).

There are some studies in Banach spaces related to these results. In [22] Takahashi, Wong and Yao introduced the generalized nonspreading mapping and the skew-generalized nonspreading mapping in a Banach space. Let $E$ be a smooth Banach space and let $C$ be a nonempty subset of $E$. A mapping $T$ from $C$ into $E$ is said to be generalized nonspreading if there exist $\alpha, \beta, \gamma, \delta, \varepsilon, \zeta \in \mathbb{R}$ such that

$$
\begin{aligned}
& \alpha \phi(T x, T y)+\beta \phi(x, T y)+\gamma \phi(T x, y)+\delta \phi(x, y) \\
& \quad \leq \varepsilon(\phi(T y, T x)-\phi(T y, x))+\zeta(\phi(y, T x)-\phi(y, x))
\end{aligned}
$$

for any $x, y \in C$, where $J$ is the duality mapping on $E$ and

$$
\phi(u, v)=\|u\|^{2}-2\langle u, J v\rangle+\|v\|^{2} .
$$

Such a mapping is said to be $(\alpha, \beta, \gamma, \delta, \varepsilon, \zeta)$-generalized nonspreading. A mapping $T$ from $C$ into $E$ is said to be skew-generalized nonspreading if there exist $\alpha, \beta, \gamma, \delta, \varepsilon, \zeta \in \mathbb{R}$ such that

$$
\begin{aligned}
& \alpha \phi(T x, T y)+\beta \phi(x, T y)+\gamma \phi(T x, y)+\delta \phi(x, y) \\
& \quad \leq \varepsilon(\phi(T y, T x)-\phi(y, T x))+\zeta(\phi(T y, x)-\phi(y, x))
\end{aligned}
$$

for any $x, y \in C$. Such a mapping is said to be $(\alpha, \beta, \gamma, \delta, \varepsilon, \zeta)$-skew-generalized nonspreading. These classes include the class of generalized hybrid mappings in a Hilbert space, however, it does not include the class of widely more generalized hybrid mappings. Moreover they introduced some extensions of attractive point and proved some attractive point theorems. $x \in E$ is an attractive point of $T$ if

$$
\phi(x, T y) \leq \phi(x, y)
$$


for any $y \in C ; x \in E$ is a skew-attractive point of $T$ if

$$
\phi(T y, x) \leq \phi(y, x)
$$

for any $y \in C$. Let

$$
\begin{aligned}
& A(T)=\{x \in E \mid \phi(x, T y) \leq \phi(x, y) \text { for any } y \in C\} \\
& B(T)=\{x \in E \mid \phi(T y, x) \leq \phi(y, x) \text { for any } y \in C\} .
\end{aligned}
$$

Let $C$ be a nonempty subset of a smooth Banach space $E$. A mapping $T$ from $C$ into $E$ is said to be generalized nonexpansive [4] if the set of all fixed points of $T$ is nonempty and

$$
\phi(T x, y) \leq \phi(x, y)
$$

for any $x \in C$ and for any fixed point $y$ of $T$.

Let $C$ be a nonempty subset of $E$ of a Banach space $E$. A mapping $R$ from $E$ onto $C$ is said to be sunny if

$$
R(R x+t(x-R x))=R x
$$

for any $x \in E$ and for any $t \in[0, \infty)$. A mapping $R$ from $E$ onto $C$ is called a retraction or a projection if $R x=x$ for any $x \in C$.

Takahashi, Wong and Yao [22] also proved the following mean convergence theorem.

Theorem 1.1. Let $E$ be a uniformly convex Banach space with a Fréchet differentiable norm. Let $C$ be a nonempty subset of $E$ and let $T$ be an $(\alpha, \beta, \gamma, \delta, \varepsilon, \zeta)$-generalized nonspreading mapping from $C$ into itself satisfying $\alpha+\beta+\gamma+\delta \geq 0$ and $\alpha+\beta>0$. Suppose that $A(T)=B(T) \neq \emptyset$. Let $R$ be the sunny generalized nonexpansive retraction of $E$ onto $B(T)$. Then, for any $x \in C$,

$$
S_{n} x \stackrel{\text { def }}{=} \frac{1}{n} \sum_{k=0}^{n-1} T^{k} x
$$

is weakly convergent to an element $q \in A(T)$, where $q=\lim _{n \rightarrow \infty} R T^{n} x$. Additionally, if $C$ is closed and convex, then $q$ is a fixed point of $T$.

On the other hand, Atsushiba, Iemoto, Kubota and Takeuchi in [1] introduced the concept of acute points as an extension of attractive points in a Hilbert space. Let $H$ be a real Hilbert space. Let $C$ be a nonempty subset of $H$ and let $T$ be a mapping from $C$ into $H$ and $k \in[0,1] . x \in H$ is called a $k$-acute point of $T$ if

$$
\|x-T y\|^{2} \leq\|x-y\|^{2}+k\|y-T y\|^{2}
$$

for any $y \in C$. Let

$$
\mathscr{A}_{k}(T)=\left\{x \in H \mid\|x-T y\|^{2} \leq\|x-y\|^{2}+k\|y-T y\|^{2} \text { for any } y \in C\right\} .
$$

Moreover, using a concept of acute points, they proved convergence theorems without convexity of set $C$.

Motivated by these results, we [11] introduced a new class of mappings on Banach spaces corresponding to the class of all widely more generalized hybrid mappings on Hilbert spaces. In this paper, we introduce some extensions of mean convergence theorems without convexity. 


\section{PRELIMINARIES}

We know that the following assertions hold; see, for instance, [19].

(T1) If a Banach space $E$ is unformly convex, then $E$ is reflexive.

(T2) Let $E$ be a Banach space, let $E^{*}$ be the topological dual space of $E$ and let $J$ be the duality mapping on $E$ defined by

$$
J(x)=\left\{x^{*} \in E^{*} \mid\|x\|^{2}=\left\langle x, x^{*}\right\rangle=\left\|x^{*}\right\|^{2}\right\}
$$

for any $x \in E$. Then $E$ is strictly convex if and only if $J$ is injective, that is, $x \neq y$ implies $J(x) \cap J(y)=\emptyset$.

(T3) Let $E$ be a Banach space, let $E^{*}$ be the topological dual space of $E$ and let $J$ be the duality mapping on $E$. Then $E$ is reflexive if and only if $J$ is surjective, that is, $\bigcup_{x \in E} J(x)=E^{*}$.

(T4) Let $E$ be a Banach space and let $J$ be the duality mapping on $E$. Then $E$ is smooth if and only if $J$ is single-valued.

(T5) Let $E$ be a Banach space and let $J$ be the duality mapping on $E$. Then

$$
\left\langle x-y, x^{*}-y^{*}\right\rangle \geq 0
$$

holds for any $x, y \in E$, for any $x^{*} \in J(x)$ and for any $y^{*} \in J(y)$.

(T6) Let $E$ be a Banach space and let $J$ be the duality mapping on $E$. If $J$ is single-valued, then $J$ is norm-to-weak* continuous.

(T7) Let $E$ be a Banach space and let $J$ be the duality mapping on $E$. If $E$ has the Fréche differentiable norm, then $J$ is norm-to-norm continuous.

(T8) Let $E$ be a Banach space and let $J$ be the duality mapping on $E$. Then $E$ is strictly convex if and only if

$$
1-\left\langle x, y^{*}\right\rangle>0
$$

for any $x, y \in E$ with $x \neq y$ and $\|x\|=\|y\|=1$ and for any $y^{*} \in J(y)$.

(T9) Let $E$ be a Banach space and let $E^{*}$ be the topological dual space of $E$. Then $E$ is reflexive if and only if $E^{*}$ is reflexive.

(T10) Let $E$ be a Banach space and let $E^{*}$ be the topological dual space of $E$. If $E^{*}$ is strictly convex, then $E$ is smooth. Conversely, $E$ is reflexive and smooth, then $E^{*}$ is strictly convex.

(T11) Let $E$ be a Banach space and let $E^{*}$ be the topological dual space of $E$. If $E^{*}$ is smooth, then $E$ is strictly convex. Conversely, $E$ is reflexive and strictly convex, then $E^{*}$ is smooth.

(T12) Let $E$ be a Banach space and let $E^{*}$ be the topological dual space of $E$. $E$ has uniformly Frécht differentiable norm if and only if $E^{*}$ is uniformly convex.

(T13) Let $E$ be a Banach space and let $E^{*}$ be the topological dual space of $E$. $E$ is strictly convex and reflexive and has Kadec-Klee property if and only if $E^{*}$ has Fréchet differentiable norm.

Let $E$ be a smooth Banach space. Let $J$ be the duality mapping on $E$ and let $\phi$ be the mapping from $E \times E$ into $[0, \infty)$ defined by

$$
\phi(x, y)=\|x\|^{2}-2\langle x, J y\rangle+\|y\|^{2}
$$

for any $x, y \in E$. Since by (T4) $J$ is single-valued, $\phi$ is well-defined. It is obvious that $x=y$ implies $\phi(x, y)=0$. Conversely, by $(\mathrm{T} 8)$ 
(T14) If $E$ is also strictly convex, then $\phi(x, y)=0$ implies $x=y$.

Let $E$ be a strictly convex and smooth Banach space. By (T2) an (T4) $J$ is a bijective mapping from $E$ onto $J(E)$. In particular, if $E$ is also reflective, then by (T3) $J$ is a bijective mapping from $E$ onto $E^{*}$. Suppose that $E$ is strictly convex, reflective and smooth. Let $\phi_{*}$ be the mapping from $E^{*} \times E^{*}$ into $[0, \infty)$ defined by

$$
\phi_{*}\left(x^{*}, y^{*}\right)=\left\|x^{*}\right\|^{2}-2\left\langle J^{-1} y^{*}, x^{*}\right\rangle+\left\|y^{*}\right\|^{2}
$$

for any $x^{*}, y^{*} \in E^{*}$. Then

$$
\phi_{*}\left(x^{*}, y^{*}\right)=\phi\left(J^{-1} y^{*}, J^{-1} x^{*}\right)
$$

holds. Therefore

$(\mathrm{T} 14)^{*} \quad \phi_{*}\left(x^{*}, y^{*}\right)=0$ if and only if $x^{*}=y^{*}$.

The following two lemmas were proved in [4].

Lemma 2.1. Let $E$ be a strictly convex and smooth Banach space and let $C$ be a nonempty closed subset of $E$. Suppose that there exists a sunny generalized nonexpansive retraction of $E$ onto $C$. Then the sunny generalized nonexpansive retraction is uniquely determined.

Lemma 2.2. Let $E$ be a strictly convex and smooth Banach space and let $C$ be a nonempty closed subset of E. Suppose that there exists a sunny generalized nonexpansive retraction of $E$ onto $C$. Then the following hold.

(i) $z=R_{C} x$ if and only if $\langle x-z, J z-J y\rangle \geq 0$ for any $y \in C$;

(ii) $\phi\left(R_{C} x, y\right)+\phi\left(x, R_{C} x\right) \leq \phi(x, y)$ for any $y \in C$.

The following two lemmas were proved in [17].

Lemma 2.3. Let $E$ be a strictly convex, reflexive and smooth Banach space and let $C$ be a nonempty closed subset of $E$. Then the following are equivalent:

(i) There exists a sunny generalized nonexpansive retraction of $E$ onto $C$;

(ii) There exists a generalized nonexpansive retraction of $E$ onto $C$;

(iii) $J(C)$ is closed and convex.

Lemma 2.4. Let $E$ be a strictly convex, reflexive and smooth Banach space. Let $C$ be a nonempty closed subset of $E$ and $(x, z) \in E \times C$. Suppose that there exists a sunny generalized nonexpansive retraction $R_{C}$ of $E$ onto $C$. Then the following are equivalent:

(i) $\quad z=R_{C} x$;

(ii) $\phi(x, z)=\min _{y \in C} \phi(x, y)$.

Lemma 2.5. [22] Let $E$ be a uniformly convex and smooth Banach space. Let $C$ be a nonempty subset of $E$. Let $T$ be a mapping from $C$ into itself with $B(T) \neq \emptyset$ and let $R$ be the sunny generalized nonexpansive retraction of $E$ onto $B(T)$. Then for any $x \in C,\left\{R T^{n} x\right\}$ is strongly convergent to an element in $B(T)$. 


\section{ACUTE POINT AND SKEW-ACUTE POINT}

Most of the results presented in this his section were stated in [11], however, we still give the proof for the sake of completeness.

Let $E$ be a smooth Banach space and let $C$ be a nonempty subset of $E$. Let $T$ be a mapping from $C$ into $E$ and let $k, \ell \in \mathbb{R} . x \in E$ is called a $(k, \ell)$-acute point of $T$ if

$$
\phi(x, T y) \leq \phi(x, y)+k \phi(y, T y)+\ell \phi(T y, y)
$$

for any $y \in C$. Recall that $x \in E$ is called a $(k, \ell)$-skew-acute point of $T$ if

$$
\phi(T y, x) \leq \phi(y, x)+k \phi(y, T y)+\ell \phi(T y, y)
$$

for any $y \in C$. Let

$$
\begin{aligned}
& \mathscr{A}_{k, \ell}(T) \\
& =\{x \in E \mid \phi(x, T y) \leq \phi(x, y)+k \phi(y, T y)+\ell \phi(T y, y) \text { for any } y \in C\} ; \\
& \mathscr{B}_{k, \ell}(T) \\
& =\{x \in E \mid \phi(T y, x) \leq \phi(y, x)+k \phi(y, T y)+\ell \phi(T y, y) \text { for any } y \in C\} .
\end{aligned}
$$

It is obvious that

$$
\mathscr{A}_{k_{1}, \ell_{1}}(T) \subset \mathscr{A}_{k_{2}, \ell_{2}}(T), \mathscr{B}_{k_{1}, \ell_{1}}(T) \subset \mathscr{B}_{k_{2}, \ell_{2}}(T)
$$

for any $k_{1}, k_{2}, \ell_{1}, \ell_{2} \in \mathbb{R}$ with $k_{1} \leq k_{2}$ and $\ell_{1} \leq \ell_{2}$.

The following lemmas are important to characterize them.

Lemma 3.1. Let $E$ be a smooth Banach space and let $C$ be a nonempty subset of $E$. Let $T$ be a mapping from $C$ into $E$ and let $k, \ell \in \mathbb{R}$. Then $\mathscr{A}_{k, \ell}(T)$ is closed and convex.

Proof. (3.1) is equivalent to

$$
2\langle x, J y-J T y\rangle \leq(k-1) \phi(y, T y)+\ell \phi(T y, y)+2\langle y, J y-J T y\rangle .
$$

Since

$$
\phi(u, v)=\phi(u, w)+\phi(w, v)+2\langle u-w, J w-J v\rangle
$$

for any $u, v, w \in E . \mathscr{A}_{k, \ell}(T)$ is closed and convex.

Lemma 3.2. Let $E$ be a smooth Banach space and let $C$ be a nonempty subset of $E$. Let $T$ be a mapping from $C$ into $E$ and let $k, \ell \in \mathbb{R}$. Then $\mathscr{B}_{k, \ell}(T)$ is closed.

Proof. (3.2) is equivalent to

$$
2\langle y-T y, J x\rangle \leq k \phi(y, T y)+(\ell-1) \phi(T y, y)+2\langle y-T y, J y\rangle
$$

from (3.3). Moreover, from (T6), one sees that $J$ is norm-to-weak* continuous. Therefore $\mathscr{B}_{k, \ell}(T)$ is closed.

Let $E^{*}$ be the dual space of a strictly convex, reflexive and smooth Banach space $E$. Let $C^{*}$ be a nonempty subset of $E^{*}$ and let $T^{*}$ be a mapping from $C^{*}$ into $E^{*}$. Let $k, \ell \in \mathbb{R} . \quad x^{*} \in E^{*}$ is called a $(k, \ell)-*$-acute point of $T^{*}$ if

$$
\phi_{*}\left(x^{*}, T^{*} y^{*}\right) \leq \phi_{*}\left(x^{*}, y^{*}\right)+k \phi_{*}\left(y^{*}, T^{*} y^{*}\right)+\ell \phi_{*}\left(T^{*} y^{*}, y^{*}\right)
$$




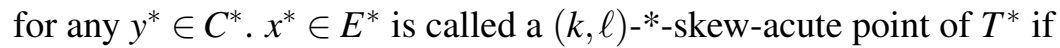

$$
\phi_{*}\left(T^{*} y^{*}, x^{*}\right) \leq \phi_{*}\left(y^{*}, x^{*}\right)+k \phi_{*}\left(y^{*}, T^{*} y^{*}\right)+\ell \phi_{*}\left(T^{*} y^{*}, y^{*}\right)
$$

for any $y^{*} \in C^{*}$. Let

$$
\begin{aligned}
& \mathscr{A}_{k, \ell}^{*}\left(T^{*}\right) \\
& =\left\{\begin{array}{l|l}
x^{*} \in E^{*} & \begin{array}{l}
\phi_{*}\left(x^{*}, T^{*} y^{*}\right) \leq \phi_{*}\left(x^{*}, y^{*}\right)+k \phi_{*}\left(y^{*}, T^{*} y^{*}\right)+\ell \phi_{*}\left(T^{*} y^{*}, y^{*}\right) \\
\text { for any } y^{*} \in C^{*}
\end{array}
\end{array}\right\} ; \\
& \mathscr{B}_{k, \ell}^{*}\left(T^{*}\right) \\
& =\left\{\begin{array}{l|l}
x^{*} \in E^{*} & \begin{array}{l}
\phi_{*}\left(T^{*} y^{*}, x^{*}\right) \leq \phi_{*}\left(y^{*}, x^{*}\right)+k \phi_{*}\left(y^{*}, T^{*} y^{*}\right)+\ell \phi_{*}\left(T^{*} y^{*}, y^{*}\right) \\
\text { for any } y^{*} \in C^{*}
\end{array}
\end{array}\right\} .
\end{aligned}
$$

Lemma 3.3. Let $E^{*}$ be the dual space of a strictly convex, reflective and smooth Banach space $E$ and let $C^{*}$ be a nonempty subset of $E^{*}$. Let $T^{*}$ be a mapping from $C^{*}$ into $E^{*}$ and let $k, \ell \in \mathbb{R}$. Then $\mathscr{A}_{k, \ell}^{*}\left(T^{*}\right)$ is closed and convex.

Proof. (3.4) is equivalent to

$$
\begin{aligned}
& 2\left\langle J^{-1} y^{*}-J^{-1} T^{*} y^{*}, x^{*}\right\rangle \\
& \quad \leq(k-1) \phi_{*}\left(y^{*}, T^{*} y^{*}\right)+\ell \phi_{*}\left(T^{*} y^{*}, y^{*}\right)+2\left\langle J^{-1} y^{*}-J^{-1} T^{*} y^{*}, y^{*}\right\rangle
\end{aligned}
$$

from (3.3) and (2.1), $\mathscr{A}_{k, \ell}^{*}\left(T^{*}\right)$ is closed and convex.

Lemma 3.4. Let $E^{*}$ be the dual space of a strictly convex, reflexive and smooth Banach space $E$, let $C^{*}$ be a nonempty subset of $E^{*}$, let $T^{*}$ be a mapping from $C^{*}$ into $E^{*}$ and let $k, \ell \in \mathbb{R}$. Then $\mathscr{B}_{k, \ell}^{*}\left(T^{*}\right)$ is closed.

Proof. (3.5) is equivalent to

$$
\begin{aligned}
& 2\left\langle J^{-1} x^{*}, y^{*}-T^{*} y^{*}\right\rangle \\
& \quad \leq k \phi_{*}\left(y^{*}, T^{*} y^{*}\right)+(\ell-1) \phi_{*}\left(T^{*} y^{*}, y^{*}\right)+2\left\langle J^{-1} y^{*}, y^{*}-T^{*} y^{*}\right\rangle
\end{aligned}
$$

from (2.1) and (3.3). Moreover, we find from (T6) that $J^{-1}$ is norm-to-weak* continuous. Therefore $\mathscr{B}_{k, \ell}^{*}\left(T^{*}\right)$ is closed.

Lemma 3.5. Let $E$ be a strictly convex, reflective and smooth Banach space and let $C$ be a nonempty subset of $E$. Let $T$ be a mapping from $C$ into $E$. Let $T^{*}=J T J^{-1}$ and let $k, \ell \in \mathbb{R}$. Then

$$
\mathscr{A}_{k, \ell}^{*}\left(T^{*}\right)=J\left(\mathscr{B}_{\ell, k}(T)\right), \mathscr{B}_{k, \ell}^{*}\left(T^{*}\right)=J\left(\mathscr{A}_{\ell, k}(T)\right) .
$$

In particular, $J\left(\mathscr{B}_{k, \ell}(T)\right)$ is closed and convex and $J\left(\mathscr{A}_{k, \ell}(T)\right)$ is closed.

Proof. Let $x^{*} \in \mathscr{A}_{k, \ell}^{*}\left(T^{*}\right)$. Then

$$
\phi_{*}\left(x^{*}, T^{*} y^{*}\right) \leq \phi_{*}\left(x^{*}, y^{*}\right)+k \phi_{*}\left(y^{*}, T^{*} y^{*}\right)+\ell \phi_{*}\left(T^{*} y^{*}, y^{*}\right)
$$

for any $y^{*} \in J(C)$. From (2.1), one has

$$
\begin{aligned}
& \phi\left(J^{-1} T^{*} y^{*}, J^{-1} x^{*}\right) \\
& \quad \leq \phi\left(J^{-1} y^{*}, J^{-1} x^{*}\right)+k \phi\left(J^{-1} T^{*} y^{*}, J^{-1} y^{*}\right)+\ell \phi\left(J^{-1} y^{*}, J^{-1} T^{*} y^{*}\right)
\end{aligned}
$$


for any $y^{*} \in J(C)$. Note that $J^{-1} T^{*}=T J^{-1}$. Putting $y=J^{-1} y^{*}$, we obtain

$$
\phi\left(T y, J^{-1} x^{*}\right) \leq \phi\left(y, J^{-1} x^{*}\right)+\ell \phi(y, T y)+k \phi(T y, y) .
$$

Therefore $J^{-1} x^{*} \in \mathscr{B}_{\ell, k}(T)$. Hence

$$
\mathscr{A}_{k, \ell}^{*}\left(T^{*}\right)=J\left(\mathscr{B}_{\ell, k}(T)\right) \text {. }
$$

$\mathscr{B}_{k, \ell}^{*}\left(T^{*}\right)=J\left(\mathscr{A}_{\ell, k}(T)\right)$ can be shown similarly. From Lemma 3.3, $J\left(\mathscr{B}_{k, \ell}(T)\right)$ is closed and convex. From Lemma 3.4, $J\left(\mathscr{A}_{k, \ell}(T)\right)$ is closed. This completes the proof.

Lemma 3.6. Let $E$ be a strictly convex and smooth Banach space and let $C$ be a nonempty subset of $E$. Let $T$ be a mapping from $C$ into $E$ and let $k, \ell \in \mathbb{R}$. Then the following hold.

(1) If $(k, \ell) \in(-\infty, 1] \times(-\infty, 0] \backslash\{(1,0)\}$, then $C \cap \mathscr{A}_{k, \ell}(T)$ is included in the set of all fixed points of $T$;

(2) If $(k, \ell) \in(-\infty, 0] \times(-\infty, 1] \backslash\{(0,1)\}$, then $C \cap \mathscr{B}_{k, \ell}(T)$ is included in the set of all fixed points of $T$.

Proof. Let $x \in C \cap \mathscr{A}_{k, \ell}(T)$. Then (3.1) holds for any $y \in C$. Putting $y=x$, we obtain

$$
(1-k) \phi(x, T x)-\ell \phi(T x, x) \leq 0 .
$$

If

$$
(k, \ell) \in(-\infty, 1] \times(-\infty, 0] \backslash\{(1,0)\},
$$

then we obtain from (T14) that $x=T x$.

Let $x \in C \cap \mathscr{B}_{k, \ell}(T)$. Then (3.2) holds for any $y \in C$. Putting $y=x$, we obtain

$$
-k \phi(x, T x)+(1-\ell) \phi(T x, x) \leq 0 .
$$

If

$$
(k, \ell) \in(-\infty, 0] \times(-\infty, 1] \backslash\{(0,1)\},
$$

then we obtain from (T14) that $x=T x$.

Lemma 3.7. Let $E^{*}$ be a strictly convex and smooth topological dual space of a Banach space and let $C^{*}$ be a nonempty subset of $E^{*}$. Let $T^{*}$ be a mapping from $C^{*}$ into $E^{*}$ and let $k, \ell \in \mathbb{R}$. Then the following hold.

(1) If $(k, \ell) \in(-\infty, 1] \times(-\infty, 0] \backslash\{(1,0)\}$, then $C \cap \mathscr{A}_{k, \ell}^{*}\left(T^{*}\right)$ is included in the set of all fixed points of $T^{*}$;

(2) If $(k, \ell) \in(-\infty, 0] \times(-\infty, 1] \backslash\{(0,1)\}$, then $C \cap \mathscr{B}_{k, \ell}^{*}\left(T^{*}\right)$ is included in the set of all fixed points of $T^{*}$.

Proof. Let $x^{*} \in C^{*} \cap \mathscr{A}_{k, \ell}^{*}\left(T^{*}\right)$. Then (3.4) holds for any $y^{*} \in C^{*}$. Putting $y^{*}=x^{*}$, we obtain

$$
(1-k) \phi_{*}\left(x^{*}, T^{*} x^{*}\right)-\ell \phi_{*}\left(T^{*} x^{*}, x^{*}\right) \leq 0 .
$$

If

$$
(k, \ell) \in(-\infty, 1] \times(-\infty, 0] \backslash\{(1,0)\},
$$

then we obtain from (T14)* that $x^{*}=T^{*} x^{*}$. 
Let $x^{*} \in C^{*} \cap \mathscr{B}_{k, \ell}^{*}\left(T^{*}\right)$. Then (3.5) holds for any $y^{*} \in C^{*}$. Putting $y^{*}=x^{*}$, by we obtain

$$
-k \phi_{*}\left(x^{*}, T^{*} x^{*}\right)+(1-\ell) \phi_{*}\left(T^{*} x^{*}, x^{*}\right) \leq 0 .
$$

If

$$
(k, \ell) \in(-\infty, 0] \times(-\infty, 1] \backslash\{(0,1)\},
$$

then we obtain from (T14)* that $x^{*}=T^{*} x^{*}$.

\section{MeAn CONVERGENCE THEOREMS}

Let $E$ be a smooth Banach space and let $C$ be a nonempty subset of $E$. A mapping $T$ from $C$ into $E$ is called a generalized pseudocontraction [11] if there exist $\alpha_{1}, \alpha_{2}, \beta_{1}, \beta_{2}, \gamma_{1}, \gamma_{2}, \delta_{1}, \delta_{2}, \varepsilon_{1}, \varepsilon_{2}, \zeta_{1}, \zeta_{2} \in \mathbb{R}$ such that

$$
\begin{aligned}
\alpha_{1} \phi & (T x, T y)+\alpha_{2} \phi(T y, T x)+\beta_{1} \phi(x, T y)+\beta_{2} \phi(T y, x) \\
& +\gamma_{1} \phi(T x, y)+\gamma_{2} \phi(y, T x)+\delta_{1} \phi(x, y)+\delta_{2} \phi(y, x) \\
& +\varepsilon_{1} \phi(T x, x)+\varepsilon_{2} \phi(x, T x)+\zeta_{1} \phi(y, T y)+\zeta_{2} \phi(T y, y) \\
\leq & 0
\end{aligned}
$$

for any $x, y \in C$. Such a mapping is called an $\left(\alpha_{1}, \alpha_{2}, \beta_{1}, \beta_{2}, \gamma_{1}, \gamma_{2}, \delta_{1}, \delta_{2}, \varepsilon_{1}, \varepsilon_{2}, \zeta_{1}, \zeta_{2}\right)$-generalized pseudocontraction.

Theorem 4.1. Let $E$ be a uniformly convex Banach space with a Fréchet differentiable norm. Let $C$ be a nonempty subset of $E$ and let $T$ be an $\left(\alpha_{1}, \alpha_{2}, \beta_{1}, \beta_{2}, \gamma_{1}, \gamma_{2}, \delta_{1}, \delta_{2}, \varepsilon_{1}, \varepsilon_{2}, \zeta_{1}, \zeta_{2}\right)$-generalized pseudocontraction from $C$ into itself. Suppose that there exists $\lambda \in[0,1]$ such that

$$
\begin{aligned}
& (1-\lambda)\left(\alpha_{1}+\beta_{1}+\gamma_{1}+\delta_{1}\right)+\lambda\left(\alpha_{2}+\beta_{2}+\gamma_{2}+\delta_{2}\right) \geq 0 \\
& \lambda\left(\alpha_{1}+\gamma_{1}\right)+(1-\lambda)\left(\alpha_{2}+\beta_{2}\right) \geq 0 \\
& \lambda\left(\beta_{1}+\delta_{1}\right)+(1-\lambda)\left(\gamma_{2}+\delta_{2}\right) \geq 0 \\
& (1-\lambda) \varepsilon_{1}+\lambda \zeta_{2} \geq 0 \\
& \lambda \zeta_{1}+(1-\lambda) \varepsilon_{2} \geq 0 \\
& (1-\lambda)\left(\alpha_{1}+\beta_{1}\right)+\lambda\left(\alpha_{2}+\gamma_{2}\right)>0
\end{aligned}
$$

and suppose that

$$
\mathscr{A}_{-\frac{(1-\lambda) \zeta_{1}+\lambda \varepsilon_{2}}{(1-\lambda)\left(\alpha_{1}+\beta_{1}\right)+\lambda\left(\alpha_{2}+\gamma_{2}\right)},-\frac{\lambda \varepsilon_{1}+(1-\lambda) \zeta_{2}}{(1-\lambda)\left(\alpha_{1}+\beta_{1}\right)+\lambda\left(\alpha_{2}+\gamma_{2}\right)}}(T) \subset B(T) \neq \emptyset .
$$

Let $R$ be the sunny generalized nonexpansive retraction of $E$ onto $B(T)$. Then, for any $x \in C$,

$$
S_{n} x \stackrel{\text { def }}{=} \frac{1}{n} \sum_{k=0}^{n-1} T^{k} x
$$

is weakly convergent to an element

$$
q \in \mathscr{A}_{-\frac{(1-\lambda) \zeta_{1}+\lambda \varepsilon_{2}}{(1-\lambda)\left(\alpha_{1}+\beta_{1}\right)+\lambda\left(\alpha_{2}+\gamma_{2}\right)},-\frac{\lambda \varepsilon_{1}+(1-\lambda) \zeta_{2}}{(1-\lambda)\left(\alpha_{1}+\beta_{1}\right)+\lambda\left(\alpha_{2}+\gamma_{2}\right)}}(T),
$$

where $q=\lim _{n \rightarrow \infty} R T^{n} x$. In addition, if $C$ is closed and convex and one of the following holds:

(1) $\quad(1-\lambda)\left(\alpha_{1}+\beta_{1}+\zeta_{1}\right)+\lambda\left(\alpha_{2}+\gamma_{2}+\varepsilon_{2}\right)>0$ and $\lambda \varepsilon_{1}+(1-\lambda) \zeta_{2} \geq 0$;

(2) $(1-\lambda)\left(\alpha_{1}+\beta_{1}+\zeta_{1}\right)+\lambda\left(\alpha_{2}+\gamma_{2}+\varepsilon_{2}\right) \geq 0$ and $\lambda \varepsilon_{1}+(1-\lambda) \zeta_{2}>0$,

then $q$ is a fixed point of $T$. 
Proof. By Lemma 3.2, we know that $B(T)$ is closed. By Lemma 3.5, we know that $J(B(T))$ is closed and convex. Using Lemmas 2.3 and 2.1, there exists a unique sunny nonexpansive retraction $R$ of $E$ onto $B(T)$. Changing the variables $x$ and $y$ in (4.1), we obtain

$$
\begin{aligned}
\alpha_{2} \phi & (T x, T y)+\alpha_{1} \phi(T y, T x)+\gamma_{2} \phi(x, T y)+\gamma_{1} \phi(T y, x) \\
& +\beta_{2} \phi(T x, y)+\beta_{1} \phi(y, T x)+\delta_{2} \phi(x, y)+\delta_{1} \phi(y, x) \\
& +\zeta_{2} \phi(T x, x)+\zeta_{1} \phi(x, T x)+\varepsilon_{2} \phi(y, T y)+\varepsilon_{1} \phi(T y, y) \\
\leq & 0 .
\end{aligned}
$$

From (4.1) and (4.2), we obtain

$$
\begin{aligned}
& \left((1-\lambda) \alpha_{1}+\lambda \alpha_{2}\right) \phi(T x, T y)+\left(\lambda \alpha_{1}+(1-\lambda) \alpha_{2}\right) \phi(T y, T x) \\
& \quad+\left((1-\lambda) \beta_{1}+\lambda \gamma_{2}\right) \phi(x, T y)+\left(\lambda \gamma_{1}+(1-\lambda) \beta_{2}\right) \phi(T y, x) \\
& \quad+\left((1-\lambda) \gamma_{1}+\lambda \beta_{2}\right) \phi(T x, y)+\left(\lambda \beta_{1}+(1-\lambda) \gamma_{2}\right) \phi(y, T x) \\
& \quad+\left((1-\lambda) \delta_{1}+\lambda \delta_{2}\right) \phi(x, y)+\left(\lambda \delta_{1}+(1-\lambda) \delta_{2}\right) \phi(y, x) \\
& \quad+\left((1-\lambda) \varepsilon_{1}+\lambda \zeta_{2}\right) \phi(T x, x)+\left(\lambda \zeta_{1}+(1-\lambda) \varepsilon_{2}\right) \phi(x, T x) \\
& \quad+\left((1-\lambda) \zeta_{1}+\lambda \varepsilon_{2}\right) \phi(y, T y)+\left(\lambda \varepsilon_{1}+(1-\lambda) \zeta_{2}\right) \phi(T y, y) \\
& \leq 0 .
\end{aligned}
$$

From (3.3), we obtain

$$
\begin{aligned}
((1- & \left.\lambda) \alpha_{1}+\lambda \alpha_{2}\right) \phi(T x, T y)+\left(\lambda \alpha_{1}+(1-\lambda) \alpha_{2}\right) \phi(T y, T x) \\
& +\left((1-\lambda) \beta_{1}+\lambda \gamma_{2}\right) \phi(x, T y)+\left(\lambda \gamma_{1}+(1-\lambda) \beta_{2}\right) \phi(T y, x) \\
& +\left((1-\lambda) \gamma_{1}+\lambda \beta_{2}\right) \phi(T x, y)+\left(\lambda \beta_{1}+(1-\lambda) \gamma_{2}\right) \phi(y, T x) \\
& +\left((1-\lambda) \delta_{1}+\lambda \delta_{2}\right) \phi(x, y)+\left(\lambda \delta_{1}+(1-\lambda) \delta_{2}\right) \phi(y, x) \\
& +\left((1-\lambda) \varepsilon_{1}+\lambda \zeta_{2}\right) \phi(T x, x)+\left(\lambda \zeta_{1}+(1-\lambda) \varepsilon_{2}\right) \phi(x, T x) \\
& +\left((1-\lambda) \zeta_{1}+\lambda \varepsilon_{2}\right) \phi(y, T y)+\left(\lambda \varepsilon_{1}+(1-\lambda) \zeta_{2}\right) \phi(T y, y) \\
= & \left((1-\lambda) \alpha_{1}+\lambda \alpha_{2}\right) \phi(T x, T y)+\left(\lambda \alpha_{1}+(1-\lambda) \alpha_{2}\right) \phi(T y, T x) \\
& -\left((1-\lambda) \alpha_{1}+\lambda \alpha_{2}\right) \phi(x, T y) \\
& +\left((1-\lambda)\left(\alpha_{1}+\beta_{1}\right)+\lambda\left(\alpha_{2}+\gamma_{2}\right)\right)(\phi(x, y)+\phi(y, T y)+2\langle x-y, J y-J T y\rangle) \\
& +\left(\lambda \gamma_{1}+(1-\lambda) \beta_{2}\right) \phi(T y, x) \\
& +\left((1-\lambda) \gamma_{1}+\lambda \beta_{2}\right) \phi(T x, y)+\left(\lambda \beta_{1}+(1-\lambda) \gamma_{2}\right) \phi(y, T x) \\
& +\left((1-\lambda) \delta_{1}+\lambda \delta_{2}\right) \phi(x, y)+\left(\lambda \delta_{1}+(1-\lambda) \delta_{2}\right) \phi(y, x) \\
& +\left((1-\lambda) \varepsilon_{1}+\lambda \zeta_{2}\right) \phi(T x, x)+\left(\lambda \zeta_{1}+(1-\lambda) \varepsilon_{2}\right) \phi(x, T x) \\
& +\left((1-\lambda) \zeta_{1}+\lambda \varepsilon_{2}\right) \phi(y, T y)+\left(\lambda \varepsilon_{1}+(1-\lambda) \zeta_{2}\right) \phi(T y, y) \\
= & \left((1-\lambda) \alpha_{1}+\lambda \alpha_{2}\right) \phi(T x, T y)+\left(\lambda \alpha_{1}+(1-\lambda) \alpha_{2}\right) \phi(T y, T x) \\
& -\left((1-\lambda) \alpha_{1}+\lambda \alpha_{2}\right) \phi(x, T y)+\left(\lambda \gamma_{1}+(1-\lambda) \beta_{2}\right) \phi(T y, x) \\
& +\left((1-\lambda) \gamma_{1}+\lambda \beta_{2}\right) \phi(T x, y)+\left(\lambda \beta_{1}+(1-\lambda) \gamma_{2}\right) \phi(y, T x) \\
& +\left((1-\lambda)\left(\alpha_{1}+\beta_{1}+\delta_{1}\right)+\lambda\left(\alpha_{2}+\gamma_{2}+\delta_{2}\right)\right) \phi(x, y) \\
& +\left(\lambda \delta_{1}+(1-\lambda) \delta_{2}\right) \phi(y, x) \\
& +\left((1-\lambda) \varepsilon_{1}+\lambda \zeta_{2}\right) \phi(T x, x)+\left(\lambda \zeta_{1}+(1-\lambda) \varepsilon_{2}\right) \phi(x, T x) \\
& +(1-\lambda)
\end{aligned}
$$




$$
\begin{aligned}
& +\left((1-\lambda)\left(\alpha_{1}+\beta_{1}+\zeta_{1}\right)+\lambda\left(\alpha_{2}+\gamma_{2}+\varepsilon_{2}\right)\right) \phi(y, T y) \\
& +\left(\lambda \varepsilon_{1}+(1-\lambda) \zeta_{2}\right) \phi(T y, y) \\
& +2\left((1-\lambda)\left(\alpha_{1}+\beta_{1}\right)+\lambda\left(\alpha_{2}+\gamma_{2}\right)\right)\langle x-y, J y-J T y\rangle .
\end{aligned}
$$

Since

$$
\begin{aligned}
& (1-\lambda)\left(\alpha_{1}+\beta_{1}+\delta_{1}\right)+\lambda\left(\alpha_{2}+\beta_{2}+\delta_{2}\right) \geq-\left((1-\lambda) \gamma_{1}+\lambda \gamma_{2}\right) ; \\
& \lambda \gamma_{1}+(1-\lambda) \beta_{2} \geq-\left(\lambda \alpha_{1}+(1-\lambda) \alpha_{2}\right) ; \\
& \lambda \delta_{1}+(1-\lambda) \delta_{2} \geq-\left(\lambda \beta_{1}+(1-\lambda) \gamma_{2}\right) ; \\
& (1-\lambda) \varepsilon_{1}+\lambda \zeta_{2} \geq 0 ; \\
& \lambda \zeta_{1}+(1-\lambda) \varepsilon_{2} \geq 0,
\end{aligned}
$$

we obtain

$$
\begin{aligned}
((1- & \left.\lambda) \alpha_{1}+\lambda \alpha_{2}\right) \phi(T x, T y)+\left(\lambda \alpha_{1}+(1-\lambda) \alpha_{2}\right) \phi(T y, T x) \\
& -\left((1-\lambda) \alpha_{1}+\lambda \alpha_{2}\right) \phi(x, T y)+\left(\lambda \gamma_{1}+(1-\lambda) \beta_{2}\right) \phi(T y, x) \\
& +\left((1-\lambda) \gamma_{1}+\lambda \beta_{2}\right) \phi(T x, y)+\left(\lambda \beta_{1}+(1-\lambda) \gamma_{2}\right) \phi(y, T x) \\
& +\left((1-\lambda)\left(\alpha_{1}+\beta_{1}+\delta_{1}\right)+\lambda\left(\alpha_{2}+\gamma_{2}+\delta_{2}\right)\right) \phi(x, y) \\
& +\left(\lambda \delta_{1}+(1-\lambda) \delta_{2}\right) \phi(y, x) \\
& +\left((1-\lambda) \varepsilon_{1}+\lambda \zeta_{2}\right) \phi(T x, x)+\left(\lambda \zeta_{1}+(1-\lambda) \varepsilon_{2}\right) \phi(x, T x) \\
& +\left((1-\lambda)\left(\alpha_{1}+\beta_{1}+\zeta_{1}\right)+\lambda\left(\alpha_{2}+\gamma_{2}+\varepsilon_{2}\right)\right) \phi(y, T y) \\
& +\left(\lambda \varepsilon_{1}+(1-\lambda) \zeta_{2}\right) \phi(T y, y) \\
& +2\left((1-\lambda)\left(\alpha_{1}+\beta_{1}\right)+\lambda\left(\alpha_{2}+\gamma_{2}\right)\right)\langle x-y, J y-J T y\rangle \\
\geq & \left((1-\lambda) \alpha_{1}+\lambda \alpha_{2}\right)(\phi(T x, T y)-\phi(x, T y)) \\
& +\left(\lambda \alpha_{1}+(1-\lambda) \alpha_{2}\right)(\phi(T y, T x)-\phi(T y, x)) \\
& +\left((1-\lambda) \gamma_{1}+\lambda \beta_{2}\right)(\phi(T x, y)-\phi(x, y)) \\
& +\left(\lambda \beta_{1}+(1-\lambda) \gamma_{2}\right)(\phi(y, T x)-\phi(y, x)) \\
& +\left((1-\lambda)\left(\alpha_{1}+\beta_{1}+\zeta_{1}\right)+\lambda\left(\alpha_{2}+\gamma_{2}+\varepsilon_{2}\right)\right) \phi(y, T y) \\
& +\left(\lambda \varepsilon_{1}+(1-\lambda) \zeta_{2}\right) \phi(T y, y) \\
& +2\left((1-\lambda)\left(\alpha_{1}+\beta_{1}\right)+\lambda\left(\alpha_{2}+\gamma_{2}\right)\right)\langle x-y, J y-J T y\rangle .
\end{aligned}
$$

It follows that

$$
\begin{aligned}
((1- & \left.\lambda) \alpha_{1}+\lambda \alpha_{2}\right)(\phi(T x, T y)-\phi(x, T y)) \\
& +\left(\lambda \alpha_{1}+(1-\lambda) \alpha_{2}\right)(\phi(T y, T x)-\phi(T y, x)) \\
& +\left((1-\lambda) \gamma_{1}+\lambda \beta_{2}\right)(\phi(T x, y)-\phi(x, y)) \\
& +\left(\lambda \beta_{1}+(1-\lambda) \gamma_{2}\right)(\phi(y, T x)-\phi(y, x)) \\
& +\left((1-\lambda)\left(\alpha_{1}+\beta_{1}+\zeta_{1}\right)+\lambda\left(\alpha_{2}+\gamma_{2}+\varepsilon_{2}\right)\right) \phi(y, T y) \\
& +\left(\lambda \varepsilon_{1}+(1-\lambda) \zeta_{2}\right) \phi(T y, y)
\end{aligned}
$$




$$
\begin{aligned}
& +2\left((1-\lambda)\left(\alpha_{1}+\beta_{1}\right)+\lambda\left(\alpha_{2}+\gamma_{2}\right)\right)\langle x-y, J y-J T y\rangle \\
\leq & 0 .
\end{aligned}
$$

Replacing $x$ by $T^{k} x$ yields that

$$
\begin{aligned}
((1- & \left.\lambda) \alpha_{1}+\lambda \alpha_{2}\right)\left(\phi\left(T^{k+1} x, T y\right)-\phi\left(T^{k} x, T y\right)\right) \\
& +\left(\lambda \alpha_{1}+(1-\lambda) \alpha_{2}\right)\left(\phi\left(T y, T^{k+1} x\right)-\phi\left(T y, T^{k} x\right)\right) \\
& +\left((1-\lambda) \gamma_{1}+\lambda \beta_{2}\right)\left(\phi\left(T^{k+1} x, y\right)-\phi\left(T^{k} x, y\right)\right) \\
& +\left(\lambda \beta_{1}+(1-\lambda) \gamma_{2}\right)\left(\phi\left(y, T^{k+1} x\right)-\phi\left(y, T^{k} x\right)\right) \\
& +\left((1-\lambda)\left(\alpha_{1}+\beta_{1}+\zeta_{1}\right)+\lambda\left(\alpha_{2}+\gamma_{2}+\varepsilon_{2}\right)\right) \phi(y, T y) \\
& +\left(\lambda \varepsilon_{1}+(1-\lambda) \zeta_{2}\right) \phi(T y, y) \\
& +2\left((1-\lambda)\left(\alpha_{1}+\beta_{1}\right)+\lambda\left(\alpha_{2}+\gamma_{2}\right)\right)\left\langle T^{k} x-y, J y-J T y\right\rangle \\
\leq & 0 .
\end{aligned}
$$

Summing up these inequalities for $k=0, \ldots, n-1$ and dividing by $n$, we have

$$
\begin{aligned}
& \frac{(1-\lambda) \alpha_{1}+\lambda \alpha_{2}}{n}\left(\phi\left(T^{n} x, T y\right)-\phi(x, T y)\right) \\
& +\frac{\lambda \alpha_{1}+(1-\lambda) \alpha_{2}}{n}\left(\phi\left(T y, T^{n} x\right)-\phi(T y, x)\right) \\
& +\frac{(1-\lambda) \gamma_{1}+\lambda \beta_{2}}{n}\left(\phi\left(T^{n} x, y\right)-\phi(x, y)\right) \\
& +\frac{\lambda \beta_{1}+(1-\lambda) \gamma_{2}}{n}\left(\phi\left(y, T^{n} x\right)-\phi(y, x)\right) \\
& +\left((1-\lambda)\left(\alpha_{1}+\beta_{1}+\zeta_{1}\right)+\lambda\left(\alpha_{2}+\gamma_{2}+\varepsilon_{2}\right)\right) \phi(y, T y) \\
& +\left(\lambda \varepsilon_{1}+(1-\lambda) \zeta_{2}\right) \phi(T y, y) \\
& +2\left((1-\lambda)\left(\alpha_{1}+\beta_{1}\right)+\lambda\left(\alpha_{2}+\gamma_{2}\right)\right)\left\langle S_{n} x-y, J y-J T y\right\rangle \\
& \leq 0 \text {. }
\end{aligned}
$$

Since $B(T) \neq \emptyset$, we obtain

$$
\phi\left(T^{n} x, y\right) \leq \phi\left(T^{n-1} x, y\right)
$$

for any $x \in C$, for any $y \in B(T)$ and for any $n \in \mathbb{N}$. Therefore $\left\{T^{n} x\right\}$ is bounded. Hence $\left\{S_{n} x\right\}$ is also bounded. Therefore there exists a subsequence $\left\{S_{n_{i}} x\right\}$ of $\left\{S_{n} x\right\}$ such that $\left\{S_{n_{i}} x\right\}$ is weakly convergent to an element $p \in E$. Replacing $n$ by $n_{i}$, we conclude

$$
\begin{aligned}
& \frac{(1-\lambda) \alpha_{1}+\lambda \alpha_{2}}{n_{i}}\left(\phi\left(T^{n_{i}} x, T y\right)-\phi(x, T y)\right) \\
& +\frac{\lambda \alpha_{1}+(1-\lambda) \alpha_{2}}{n_{i}}\left(\phi\left(T y, T^{n_{i}} x\right)-\phi(T y, x)\right) \\
& \quad+\frac{(1-\lambda) \gamma_{1}+\lambda \beta_{2}}{n_{i}}\left(\phi\left(T^{n_{i}} x, y\right)-\phi(x, y)\right) \\
& \quad+\frac{\lambda \beta_{1}+(1-\lambda) \gamma_{2}}{n_{i}}\left(\phi\left(y, T^{n_{i}} x\right)-\phi(y, x)\right) \\
& +\left((1-\lambda)\left(\alpha_{1}+\beta_{1}+\zeta_{1}\right)+\lambda\left(\alpha_{2}+\gamma_{2}+\varepsilon_{2}\right)\right) \phi(y, T y)
\end{aligned}
$$




$$
\begin{aligned}
& +\left(\lambda \varepsilon_{1}+(1-\lambda) \zeta_{2}\right) \phi(T y, y) \\
& +2\left((1-\lambda)\left(\alpha_{1}+\beta_{1}\right)+\lambda\left(\alpha_{2}+\gamma_{2}\right)\right)\left\langle S_{n_{i}} x-y, J y-J T y\right\rangle \\
\leq & 0 .
\end{aligned}
$$

Putting $i \rightarrow \infty$, we obtain

$$
\begin{aligned}
& \left((1-\lambda)\left(\alpha_{1}+\beta_{1}+\zeta_{1}\right)+\lambda\left(\alpha_{2}+\gamma_{2}+\varepsilon_{2}\right)\right) \phi(y, T y)+\left(\lambda \varepsilon_{1}+(1-\lambda) \zeta_{2}\right) \phi(T y, y) \\
& \quad+2\left((1-\lambda)\left(\alpha_{1}+\beta_{1}\right)+\lambda\left(\alpha_{2}+\gamma_{2}\right)\right)\langle p-y, J y-J T y\rangle \\
& \quad \leq 0 .
\end{aligned}
$$

It follows from (3.3) that

$$
\begin{aligned}
& \left((1-\lambda) \zeta_{1}+\lambda \varepsilon_{2}\right) \phi(y, T y)+\left(\lambda \varepsilon_{1}+(1-\lambda) \zeta_{2}\right) \phi(T y, y) \\
& \quad+\left((1-\lambda)\left(\alpha_{1}+\beta_{1}\right)+\lambda\left(\alpha_{2}+\gamma_{2}\right)\right)(\phi(p, T y)-\phi(p, y)) \\
& \quad \leq 0 .
\end{aligned}
$$

Since

$$
(1-\lambda)\left(\alpha_{1}+\beta_{1}\right)+\lambda\left(\alpha_{2}+\gamma_{2}\right)>0
$$

we find

$$
\begin{aligned}
\phi(p, T y) \leq \phi(p, y) & -\frac{(1-\lambda) \zeta_{1}+\lambda \varepsilon_{2}}{(1-\lambda)\left(\alpha_{1}+\beta_{1}\right)+\lambda\left(\alpha_{2}+\gamma_{2}\right)} \phi(y, T y) \\
& -\frac{\lambda \varepsilon_{1}+(1-\lambda) \zeta_{2}}{(1-\lambda)\left(\alpha_{1}+\beta_{1}\right)+\lambda\left(\alpha_{2}+\gamma_{2}\right)} \phi(T y, y) .
\end{aligned}
$$

It follows that

$$
p \in \mathscr{A}_{-\frac{(1-\lambda) \zeta_{1}+\lambda \varepsilon_{2}}{(1-\lambda)\left(\alpha_{1}+\beta_{1}\right)+\lambda\left(\alpha_{2}+\gamma_{2}\right)},-\frac{\lambda \varepsilon_{1}+(1-\lambda) \zeta_{2}}{(1-\lambda)\left(\alpha_{1}+\beta_{1}\right)+\lambda\left(\alpha_{2}+\gamma_{2}\right)}}(T) .
$$

Using Lemma 2.2, we obtain

$$
\left\langle T^{k} x-R T^{k} x, J y-J R T^{k} x\right\rangle \leq 0
$$

for any $y \in B(T)$. By Lemma 2.5, $\left\{R T^{n} x\right\}$ is strongly convergent to an element in $B(T)$ for any $x \in C$. Let $q=\lim _{n \rightarrow \infty} R T^{n} x$. Note that $\left\{T^{n} x\right\}$ is bounded. By Lemma 2.2, $\left\{R T^{n} x\right\}$ is also bounded. Putting

$$
K=\max _{n \in \mathbb{N} \cup\{0\}, x \in C}\left\|T^{n} x-R T^{n} x\right\|,
$$

we obtain

$$
\begin{aligned}
\left\langle T^{k} x-R T^{k} x, J y-J q\right\rangle & \leq\left\langle T^{k} x-R T^{k} x, J R T^{k} x-J q\right\rangle \\
& \leq\left\|T^{k} x-R T^{k} x\right\| \cdot\left\|J R T^{k} x-J q\right\| \\
& \leq K\left\|J R T^{k} x-J q\right\| .
\end{aligned}
$$

Summing up these inequalities for $k=0, \ldots, n-1$ and dividing by $n$, we arrive at

$$
\left\langle S_{n} x-\frac{1}{n} \sum_{k=0}^{n-1} R T^{k} x, J y-J q\right\rangle \leq \frac{K}{n} \sum_{k=0}^{n-1}\left\|J R T^{k} x-J q\right\| .
$$

Since $\left\{S_{n_{i}} x\right\}$ is weakly convergent to $p$ and $J$ is norm-to-norm continuous, we obtain

$$
\langle p-q, J y-J q\rangle \leq 0 .
$$


Note that

$$
\mathscr{A}_{-\frac{(1-\lambda) \zeta_{1}+\lambda \varepsilon_{2}}{(1-\lambda)\left(\alpha_{1}+\beta_{1}\right)+\lambda\left(\alpha_{2}+\gamma_{2}\right)},-\frac{\lambda \varepsilon_{1}+(1-\lambda) \zeta_{2}}{(1-\lambda)\left(\alpha_{1}+\beta_{1}\right)+\lambda\left(\alpha_{2}+\gamma_{2}\right)}}(T) \subset B(T) .
$$

Putting $y=p$, we obtain from (3.3) that

$$
\begin{aligned}
0 & \leq 2\langle p-q, J q-J p\rangle \\
& =-\phi(p, q)-\phi(q, p) .
\end{aligned}
$$

From (T14), we have $p=q$. Therefore $\left\{S_{n} x\right\}$ is weakly convergent to $q$. In addition, if $C$ is closed and convex and (1) or (2) holds, then $\left\{S_{n} x\right\} \subset C$. Hence $q \in C$. By Lemma 3.6, we conclude that $q$ is a fixed point of $T$.

Let $E^{*}$ be the dual space of a strictly convex, reflexive and smooth Banach space $E$ and let $C^{*}$ be a nonempty subset of $E^{*}$. A mapping $T^{*}$ from $C^{*}$ into $E^{*}$ is called a *-generalized pseudocontraction [11] if there exist $\alpha_{1}, \alpha_{2}, \beta_{1}, \beta_{2}, \gamma_{1}, \gamma_{2}, \delta_{1}, \delta_{2}, \varepsilon_{1}, \varepsilon_{2}, \zeta_{1}, \zeta_{2} \in \mathbb{R}$ such that

$$
\begin{aligned}
& \alpha_{1} \phi_{*}\left(T^{*} x^{*}, T^{*} y^{*}\right)+\alpha_{2} \phi_{*}\left(T^{*} y^{*}, T^{*} x^{*}\right)+\beta_{1} \phi_{*}\left(x^{*}, T^{*} y^{*}\right)+\beta_{2} \phi_{*}\left(T^{*} y^{*}, x^{*}\right) \\
& \quad+\gamma_{1} \phi_{*}\left(T^{*} x^{*}, y^{*}\right)+\gamma_{2} \phi_{*}\left(y^{*}, T^{*} x^{*}\right)+\delta_{1} \phi_{*}\left(x^{*}, y^{*}\right)+\delta_{2} \phi_{*}\left(y^{*}, x^{*}\right) \\
& \quad+\varepsilon_{1} \phi_{*}\left(T^{*} x^{*}, x^{*}\right)+\varepsilon_{2} \phi_{*}\left(x^{*}, T^{*} x^{*}\right)+\zeta_{1} \phi_{*}\left(y^{*}, T^{*} y^{*}\right)+\zeta_{2} \phi_{*}\left(T^{*} y^{*}, y^{*}\right) \\
& \leq 0
\end{aligned}
$$

for any $x^{*}, y^{*} \in C^{*}$. Such a mapping is called an $\left(\alpha_{1}, \alpha_{2}, \beta_{1}, \beta_{2}, \gamma_{1}, \gamma_{2}, \delta_{1}, \delta_{2}, \varepsilon_{1}, \varepsilon_{2}, \zeta_{1}, \zeta_{2}\right)$-* $^{*}$ generalized pseudocontraction.

Theorem 4.2. Let $E^{*}$ be a uniformly convex topological dual space with a Fréchet differentiable norm. Let $C^{*}$ be a nonempty subset of $E^{*}$ and let $T^{*}$ be an $\left(\alpha_{1}, \alpha_{2}, \beta_{1}, \beta_{2}, \gamma_{1}, \gamma_{2}, \delta_{1}, \delta_{2}, \varepsilon_{1}, \varepsilon_{2}, \zeta_{1}, \zeta_{2}\right)-*_{-}$ generalized pseudocontraction from $C^{*}$ into itself. Suppose that there exists $\lambda \in[0,1]$ such that

$$
\begin{aligned}
& (1-\lambda)\left(\alpha_{1}+\beta_{1}+\gamma_{1}+\delta_{1}\right)+\lambda\left(\alpha_{2}+\beta_{2}+\gamma_{2}+\delta_{2}\right) \geq 0 \\
& \lambda\left(\alpha_{1}+\gamma_{1}\right)+(1-\lambda)\left(\alpha_{2}+\beta_{2}\right) \geq 0 \\
& \lambda\left(\beta_{1}+\delta_{1}\right)+(1-\lambda)\left(\gamma_{2}+\delta_{2}\right) \geq 0 \\
& (1-\lambda) \varepsilon_{1}+\lambda \zeta_{2} \geq 0 \\
& \lambda \zeta_{1}+(1-\lambda) \varepsilon_{2} \geq 0 \\
& (1-\lambda)\left(\alpha_{1}+\beta_{1}\right)+\lambda\left(\alpha_{2}+\gamma_{2}\right)>0
\end{aligned}
$$

and suppose that

$$
\mathscr{A}_{-\frac{(1-\lambda) \xi_{1}+\lambda \varepsilon_{2}}{(1-\lambda)\left(\alpha_{1}+\beta_{1}\right)+\lambda\left(\alpha_{2}+\gamma_{2}\right)}},-\frac{\lambda \varepsilon_{1}+(1-\lambda) \xi_{2}}{(1-\lambda)\left(\alpha_{1}+\beta_{1}\right)+\lambda\left(\alpha_{2}+\gamma_{2}\right)}\left(T^{*}\right) \subset \mathscr{B}_{0,0}^{*}\left(T^{*}\right) \neq \emptyset .
$$

Let $R^{*}$ be the sunny generalized nonexpansive retraction of $E^{*}$ onto $\mathscr{B}_{0,0}^{*}\left(T^{*}\right)$. Then, for any $x^{*} \in C^{*}$,

$$
S_{n}^{*} x^{*} \stackrel{\text { def }}{=} \frac{1}{n} \sum_{k=0}^{n-1}\left(T^{*}\right)^{k} x^{*}
$$

is weakly convergent to an element

$$
q^{*} \in \mathscr{A}_{-\frac{(1-\lambda) \zeta_{1}+\lambda \varepsilon_{2}}{(1-\lambda)\left(\alpha_{1}+\beta_{1}\right)+\lambda\left(\alpha_{2}+\gamma_{2}\right)},-\frac{\lambda \varepsilon_{1}+(1-\lambda) \zeta_{2}}{(1-\lambda)\left(\alpha_{1}+\beta_{1}\right)+\lambda\left(\alpha_{2}+\gamma_{2}\right)}}\left(T^{*}\right)
$$

where $q^{*}=\lim _{n \rightarrow \infty} R^{*}\left(T^{*}\right)^{n} x^{*}$. In addition, if $C^{*}$ is closed and convex and one of the following holds: 
(1) $\quad(1-\lambda)\left(\alpha_{1}+\beta_{1}+\zeta_{1}\right)+\lambda\left(\alpha_{2}+\gamma_{2}+\varepsilon_{2}\right)>0$ and $\lambda \varepsilon_{1}+(1-\lambda) \zeta_{2} \geq 0$;

(2) $(1-\lambda)\left(\alpha_{1}+\beta_{1}+\zeta_{1}\right)+\lambda\left(\alpha_{2}+\gamma_{2}+\varepsilon_{2}\right) \geq 0$ and $\lambda \varepsilon_{1}+(1-\lambda) \zeta_{2}>0$,

then $q^{*}$ is a fixed point of $T^{*}$.

Proof. By (T12) and (T13), $E$ is a strictly convex, reflexive and smooth Banach space. Therefore $\phi_{*}$ is well-defined. From (2.1) and (3.3), we obtain

$$
\phi_{*}\left(u^{*}, v^{*}\right)=\phi_{*}\left(u^{*}, w^{*}\right)+\phi_{*}\left(w^{*}, v^{*}\right)+2\left\langle J^{-1} w^{*}-J^{-1} v^{*}, u^{*}-w^{*}\right\rangle .
$$

In a similar way, one has

$$
\begin{aligned}
& \left((1-\lambda)\left(\alpha_{1}+\beta_{1}+\zeta_{1}\right)+\lambda\left(\alpha_{2}+\gamma_{2}+\varepsilon_{2}\right)\right) \phi_{*}\left(y^{*}, T^{*} y^{*}\right) \\
& \quad+\left(\lambda \varepsilon_{1}+(1-\lambda) \zeta_{2}\right) \phi_{*}\left(T^{*} y^{*}, y^{*}\right) \\
& \quad+2\left((1-\lambda)\left(\alpha_{1}+\beta_{1}\right)+\lambda\left(\alpha_{2}+\gamma_{2}\right)\right)\left\langle J^{-1} y^{*}-J^{-1} T^{*} y^{*}, p^{*}-y^{*}\right\rangle \\
& \quad \leq 0
\end{aligned}
$$

for any $x^{*}, y^{*} \in C^{*}$, where $p^{*} \in E^{*}$ is a weak limit of a subsequence $\left\{S_{n_{i}}^{*} x^{*}\right\}$ of $\left\{S_{n}^{*} x^{*}\right\}$. From (4.3), we obtain

$$
\begin{aligned}
& \left((1-\lambda) \zeta_{1}+\lambda \varepsilon_{2}\right) \phi_{*}\left(y^{*}, T^{*} y^{*}\right)+\left(\lambda \varepsilon_{1}+(1-\lambda) \zeta_{2}\right) \phi_{*}\left(T^{*} y^{*}, y^{*}\right) \\
& \quad+\left((1-\lambda)\left(\alpha_{1}+\beta_{1}\right)+\lambda\left(\alpha_{2}+\gamma_{2}\right)\right)\left(\phi_{*}\left(p^{*}, T^{*} y^{*}\right)-\phi_{*}\left(p^{*}, y^{*}\right)\right) \\
& \quad \leq 0 .
\end{aligned}
$$

Since $(1-\lambda)\left(\alpha_{1}+\beta_{1}\right)+\lambda\left(\alpha_{2}+\gamma_{2}\right)>0$, we have

$$
\begin{gathered}
\phi_{*}\left(p^{*}, T^{*} y^{*}\right) \leq \phi_{*}\left(p^{*}, y^{*}\right)-\frac{(1-\lambda) \zeta_{1}+\lambda \varepsilon_{2}}{(1-\lambda)\left(\alpha_{1}+\beta_{1}\right)+\lambda\left(\alpha_{2}+\gamma_{2}\right)} \phi_{*}\left(y^{*}, T^{*} y^{*}\right) \\
-\frac{\lambda \varepsilon_{1}+(1-\lambda) \zeta_{2}}{(1-\lambda)\left(\alpha_{1}+\beta_{1}\right)+\lambda\left(\alpha_{2}+\gamma_{2}\right)} \phi_{*}\left(T^{*} y^{*} y^{*}\right) .
\end{gathered}
$$

It follows that

$$
p^{*} \in \mathscr{A}_{-\frac{(1-\lambda) \zeta_{1}+\lambda \varepsilon_{2}}{(1-\lambda)\left(\alpha_{1}+\beta_{1}\right)+\lambda\left(\alpha_{2}+\gamma_{2}\right)},-\frac{\lambda \varepsilon_{1}+(1-\lambda) \zeta_{2}}{(1-\lambda)\left(\alpha_{1}+\beta_{1}\right)+\lambda\left(\alpha_{2}+\gamma_{2}\right)}}^{*}\left(T^{*}\right) .
$$

Using Lemma 2.2, we obtain

$$
\left\langle J^{-1} y^{*}-J^{-1} R^{*}\left(T^{*}\right)^{k} x^{*},\left(T^{*}\right)^{k} x^{*}-R^{*}\left(T^{*}\right)^{k} x^{*}\right\rangle \leq 0
$$

for any $y^{*} \in \mathscr{B}_{0,0}^{*}\left(T^{*}\right)$. By Lemma $2.5,\left\{R^{*}\left(T^{*}\right)^{n} x^{*}\right\}$ is strongly convergent to an element in $\mathscr{B}_{0,0}^{*}\left(T^{*}\right)$ for any $x^{*} \in C^{*}$. Putting $q^{*}=\lim _{n \rightarrow \infty} R^{*}\left(T^{*}\right)^{n} x^{*}$ and

$$
K=\max _{n \in \mathbb{N} \cup\{0\}, x^{*} \in C^{*}}\left\|\left(T^{*}\right)^{n} x^{*}-R^{*}\left(T^{*}\right)^{n} x^{*}\right\|,
$$

we obtain

$$
\begin{aligned}
& \left\langle J^{-1} y^{*}-J^{-1} q^{*},\left(T^{*}\right)^{k} x^{*}-R^{*}\left(T^{*}\right)^{k} x^{*}\right\rangle \\
& \quad \leq\left\langle J^{-1} R^{*}\left(T^{*}\right)^{k} x^{*}-J^{-1} q^{*},\left(T^{*}\right)^{k} x^{*}-R^{*}\left(T^{*}\right)^{k} x^{*}\right\rangle \\
& \quad \leq\left\|J^{-1} R^{*}\left(T^{*}\right)^{k} x^{*}-J^{-1} q^{*}\right\| \cdot\left\|\left(T^{*}\right)^{k} x^{*}-R^{*}\left(T^{*}\right)^{k} x^{*}\right\| \\
& \quad \leq K\left\|J^{-1} R^{*}\left(T^{*}\right)^{k} x^{*}-J^{-1} q^{*}\right\| .
\end{aligned}
$$


Summing up these inequalities for $k=0, \ldots, n-1$ and dividing by $n$, we obtain

$$
\left\langle J^{-1} y^{*}-J^{-1} q^{*}, S_{n}^{*} x^{*}-\frac{1}{n} \sum_{k=0}^{n-1} R^{*}\left(T^{*}\right)^{k} x^{*}\right\rangle \leq \frac{K}{n} \sum_{k=0}^{n-1}\left\|J^{-1} R^{*}\left(T^{*}\right)^{k} x^{*}-J^{-1} q^{*}\right\| .
$$

Since $\left\{S_{n_{i}}^{*} x^{*}\right\}$ is weakly convergent to $p^{*}$ and $J^{-1}$ is norm-to-norm continuous, we obtain

$$
\left\langle J^{-1} y^{*}-J^{-1} q^{*}, p^{*}-q^{*}\right\rangle \leq 0 .
$$

Note that

$$
\mathscr{A}_{-\frac{(1-\lambda) \zeta_{1}+\lambda \varepsilon_{2}}{(1-\lambda)\left(\alpha_{1}+\beta_{1}\right)+\lambda\left(\alpha_{2}+\gamma_{2}\right)},-\frac{\lambda \varepsilon_{1}+(1-\lambda) \zeta_{2}}{(1-\lambda)\left(\alpha_{1}+\beta_{1}\right)+\lambda\left(\alpha_{2}+\gamma_{2}\right)}}\left(T^{*}\right) \subset \mathscr{B}_{0,0}^{*}\left(T^{*}\right) .
$$

Putting $y^{*}=p^{*}$, we obtain from (3.3) that

$$
\begin{aligned}
0 & \leq 2\left\langle J^{-1} y^{*}-J^{-1} q^{*}, p^{*}-q^{*}\right\rangle \\
& =-\phi_{*}\left(p^{*}, q^{*}\right)-\phi_{*}\left(q^{*}, p^{*}\right) .
\end{aligned}
$$

From (T14)*, we obtain $p^{*}=q^{*}$. Therefore $\left\{S_{n}^{*} x^{*}\right\}$ is weakly convergent to $q^{*}$. In addition, if $C^{*}$ is closed and convex and (1) or (2) holds, then $\left\{S_{n}^{*} x^{*}\right\} \subset C^{*}$. Hence $q^{*} \in C^{*}$. By Lemma 3.7, we conclude that $q^{*}$ is a fixed point of $T^{*}$.

By Theorem 4.2, we can obtain the following result immediately.

Theorem 4.3. Let $E$ be a strictly convex and reflexive Banach space with Kadec-Klee property and $a$ uniformly Fréchet differentiable norm. Let $C$ be a nonempty subset of $E$ and let $T$ be an $\left(\alpha_{1}, \alpha_{2}, \beta_{1}\right.$, $\left.\beta_{2}, \gamma_{1}, \gamma_{2}, \delta_{1}, \delta_{2}, \varepsilon_{1}, \varepsilon_{2}, \zeta_{1}, \zeta_{2}\right)$-generalized pseudocontraction from $C$ into itself. Suppose that there exists $\lambda \in[0,1]$ such that

$$
\begin{aligned}
& (1-\lambda)\left(\alpha_{2}+\beta_{2}+\gamma_{2}+\delta_{2}\right)+\lambda\left(\alpha_{1}+\beta_{1}+\gamma_{1}+\delta_{1}\right) \geq 0 \\
& \lambda\left(\alpha_{2}+\gamma_{2}\right)+(1-\lambda)\left(\alpha_{1}+\beta_{1}\right) \geq 0 \\
& \lambda\left(\beta_{2}+\delta_{2}\right)+(1-\lambda)\left(\gamma_{1}+\delta_{1}\right) \geq 0 \\
& (1-\lambda) \varepsilon_{2}+\lambda \zeta_{1} \geq 0 \\
& \lambda \zeta_{2}+(1-\lambda) \varepsilon_{1} \geq 0 \\
& (1-\lambda)\left(\alpha_{2}+\beta_{2}\right)+\lambda\left(\alpha_{1}+\gamma_{1}\right)>0
\end{aligned}
$$

suppose that

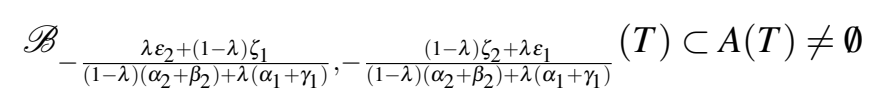

and suppose that $\mathrm{J}^{-1}$ is weakly sequentially continuous. Let $R^{*}$ be the sunny generalized nonexpansive retraction of $E^{*}$ onto $J(A(T))$. Then, for any $x \in C$,

$$
S_{n} x \stackrel{\text { def }}{=} J^{-1}\left(\frac{1}{n} \sum_{k=0}^{n-1} J T^{k} x\right)
$$

is weakly convergent to an element

$$
q \in \mathscr{B}_{-\frac{\lambda \varepsilon_{2}+(1-\lambda) \zeta_{1}}{(1-\lambda)\left(\alpha_{2}+\beta_{2}\right)+\lambda\left(\alpha_{1}+\gamma_{1}\right)},-\frac{(1-\lambda) \zeta_{2}+\lambda \varepsilon_{1}}{(1-\lambda)\left(\alpha_{2}+\beta_{2}\right)+\lambda\left(\alpha_{1}+\gamma_{1}\right)}}(T),
$$

where $q=\lim _{n \rightarrow \infty} J^{-1} R^{*} J T^{n} x$. In addition, if $J(C)$ is closed and convex and one of the following holds: 
(1) $\quad(1-\lambda)\left(\alpha_{2}+\beta_{2}+\zeta_{2}\right)+\lambda\left(\alpha_{1}+\gamma_{1}+\varepsilon_{1}\right)>0$ and $\lambda \varepsilon_{2}+(1-\lambda) \zeta_{1} \geq 0$

(2) $(1-\lambda)\left(\alpha_{2}+\beta_{2}+\zeta_{2}\right)+\lambda\left(\alpha_{1}+\gamma_{1}+\varepsilon_{1}\right) \geq 0$ and $\lambda \varepsilon_{2}+(1-\lambda) \zeta_{1}>0$,

then $q$ is a fixed point of $T$.

Proof. By (T12) and (T13), $E^{*}$ is uniformly convex with a Fréchet differentiable norm. Let $T^{*}=J T J^{-1}$. Then $T^{*}$ is a mapping from $J(C)$ into itself. Putting $x^{*}=J x$ and $y^{*}=J y$, one sees from (2.1) that (4.1) is equivalent to

$$
\begin{aligned}
\alpha_{2} \phi_{*} & \left(T^{*} x^{*}, T^{*} y^{*}\right)+\alpha_{1} \phi_{*}\left(T^{*} y^{*}, T^{*} x^{*}\right)+\beta_{2} \phi_{*}\left(x^{*}, T^{*} y^{*}\right)+\beta_{1} \phi_{*}\left(T^{*} y^{*}, x^{*}\right) \\
& \left.+\gamma_{2} \phi_{*}\left(T^{*} x^{*}, y^{*}\right)+\gamma_{1} \phi_{*}\left(y^{*}, T^{*} x^{*}\right)\right)+\delta_{2} \phi_{*}\left(x^{*}, y^{*}\right)+\delta_{1} \phi_{*}\left(y^{*}, x^{*}\right. \\
& +\varepsilon_{2} \phi_{*}\left(T^{*} x^{*}, x^{*}\right)+\varepsilon_{1} \phi_{*}\left(x^{*}, T^{*} x^{*}\right)+\zeta_{2} \phi_{*}\left(y^{*}, T^{*} y^{*}\right)+\zeta_{1} \phi_{*}\left(T^{*} y^{*}, y^{*}\right) \\
\leq & 0 .
\end{aligned}
$$

Therefore $T^{*}$ is an $\left(\alpha_{2}, \alpha_{1}, \beta_{2}, \beta_{1}, \gamma_{2}, \gamma_{1}, \delta_{2}, \delta_{1}, \varepsilon_{2}, \varepsilon_{1}, \zeta_{2}, \zeta_{1}\right)-*$-generalized pseudocontraction from $J(C)$ into itself. Since $\left(T^{*}\right)^{n} x^{*}=J T^{n} x,\left\|\left(T^{*}\right)^{n} x^{*}\right\|=\left\|J T^{n} x\right\|=\left\|T^{n} x\right\|$, one concludes that $\left\{\left(T^{*}\right)^{n} x^{*} \mid n \in\right.$ $\mathbb{N} \cup\{0\}\}$ is bounded. By Lemma 3.5, one has

$$
\begin{aligned}
& \mathscr{A}^{*} \frac{(1-\lambda) \zeta_{2}+\lambda \varepsilon_{1}}{(1-\lambda)\left(\alpha_{2}+\beta_{2}\right)+\lambda\left(\alpha_{1}+\gamma_{1}\right)},-\frac{\lambda \varepsilon_{2}+(1-\lambda) \zeta_{1}}{(1-\lambda)\left(\alpha_{2}+\beta_{2}\right)+\lambda\left(\alpha_{2}+\gamma_{2}\right)}\left(T^{*}\right) \\
& =J\left(\mathscr{B}_{-\frac{\lambda \varepsilon_{2}+(1-\lambda) \zeta_{1}}{(1-\lambda)\left(\alpha_{2}+\beta_{2}\right)+\lambda\left(\alpha_{2}+\gamma_{2}\right)},-\frac{(1-\lambda) \zeta_{2}+\lambda \varepsilon_{1}}{(1-\lambda)\left(\alpha_{2}+\beta_{2}\right)+\lambda\left(\alpha_{1}+\gamma_{1}\right)}}(T)\right), \\
& B_{0,0}^{*}\left(T^{*}\right)=J(A(T)) \text {. }
\end{aligned}
$$

From Theorem 4.2, we find that, for any $x \in C$,

$$
S_{n}^{*} x^{*}=\frac{1}{n} \sum_{k=0}^{n-1}\left(T^{*}\right)^{k} x^{*}
$$

is weakly convergent to an element

$$
q^{*} \in J\left(\mathscr{B}_{-\frac{\lambda \varepsilon_{2}+(1-\lambda) \zeta_{1}}{(1-\lambda)\left(\alpha_{2}+\beta_{2}\right)+\lambda\left(\alpha_{2}+\gamma_{2}\right)},-\frac{(1-\lambda) \zeta_{2}+\lambda \varepsilon_{1}}{(1-\lambda)\left(\alpha_{2}+\beta_{2}\right)+\lambda\left(\alpha_{1}+\gamma_{1}\right)}}(T)\right),
$$

where $q^{*}=\lim _{n \rightarrow \infty} R^{*} J T^{n} x$. Since $J^{-1}$ is weakly sequentially continuous and $J^{-1}$ is norm-to-norm continuous, one has

$$
S_{n} x=J^{-1} S_{n}^{*} J x=J^{-1}\left(\frac{1}{n} \sum_{k=0}^{n-1} J T^{k} x\right)
$$

is weakly convergent to the element

$$
q=J^{-1} q^{*} \in \mathscr{B}_{-\frac{\lambda \varepsilon_{2}+(1-\lambda) \zeta_{1}}{(1-\lambda)\left(\alpha_{2}+\beta_{2}\right)+\lambda\left(\alpha_{2}+\gamma_{2}\right)},-\frac{(1-\lambda) \zeta_{2}+\lambda \varepsilon_{1}}{(1-\lambda)\left(\alpha_{2}+\beta_{2}\right)+\lambda\left(\alpha_{1}+\gamma_{1}\right)}}(T),
$$

where $q=\lim _{n \rightarrow \infty} J^{-1} R^{*} J T^{n} x$. In addition, if $J(C)$ is closed and convex and (1) or (2) holds, then $q^{*}$ is a fixed point of $T^{*}$. Hence $q=J^{-1} q^{*}$ is a fixed point of $T$.

\section{REFERENCES}

[1] S. Atsushiba, S. Iemoto, R. Kubota, Y. Takeuchi, Convergence theorems for some classes of nonlinear mappings in Hilbert spaces, Linear Nonlinear Anal. 2 (2016), 125-153.

[2] J.-B. Baillon, Un théorème de type ergodique pour les contractions non linéaires dans un espace de Hilbert Comptes Rendus Hebdomadaires des Séances de l'Académie des Sciences. Séries A et B 280 (1975), 1511-1514. 
[3] F.E. Browder, W.V. Petryshyn, Construction of fixed points of nonlinear mappings in Hilbert space, J. Math. Anal. Appl. 20 (1967), 197-228.

[4] T. Ibaraki, W. Takahashi, A new projection and convergence theorems for the projections in Banach spaces, J. Approx. Theory 149 (2007), 1-14.

[5] T. Kawasaki, An extension of existence and mean approximation of fixed points of generalized hybrid non-self mappings in Hilbert spaces, Kyoto University Research Information Repository 1906 (2014), 13-23.

[6] T. Kawasaki, Fixed points theorems and mean convergence theorems for generalized hybrid self mappings and non-self map- pings in Hilbert spaces, Pacific J. Optim. 12 (2016), 133-150.

[7] T. Kawasaki, Fixed point theorem for widely more generalized hybrid demicontinuous mappings in Hilbert spaces, Proceedings of Nonlinear Analysis and Convex Analysis, Yokohama Publishers, Yokohama, to appear.

[8] T. Kawasaki, Fixed point theorems for widely more generalized hybrid mappings in metric spaces, Banach spaces and Hilbert spaces, J. Nonlinear Convex Anal. 19 (2018), 1675-1683.

[9] T. Kawasaki, On convergence of orbits to a fixed point for widely more generalized hybrid mappings, Nihonkai Math. J. 27 (2016), 89-97.

[10] T. Kawasaki, Fixed point theorems for widely more generalized hybrid mappings in a metric space, a Banach space and a Hilbert space, Proceedings of Nonlinear Analysis and Convex Analysis, Yokohama Publishers, Yokohama, to appear.

[11] T. Kawasaki, Fixed point and acute point theorems for new mappings in a Banach space, submitted.

[12] T. Kawasaki, T. Kobayashi, Existence and mean approximation of fixed points of generalized hybrid non-self mappings in Hilbert spaces, Sci. Math. Jpn. 77 (2014), 29-42.

[13] T. Kawasaki, W. Takahashi, Existence and mean approximation of fixed points of generalized hybrid mappings in Hilbert spaces, J. Nonlinear Convex Anal. 14 (2013), 71-87.

[14] T. Kawasaki, W. Takahashi, Fixed point and nonlinear ergodic theorems for widely more generalized hybrid mappings in Hilbert spaces and applications, Proceedings of Nonlinear Analysis and Convex Analysis, Yokohama Publishers, Yokohama, to appear.

[15] T. Kawasaki, W. Takahashi, Fixed point theorems for generalized hybrid demicontinuous mappings in Hilbert spaces, Linear Nonlinear Anal. 1 (2015), 125-138.

[16] P. Kocourek, W. Takahashi, J.-C. Yao, Fixed point theorems and weak convergence theorems for generalized hybrid mappings in Hilbert spaces, Taiwanese J. Math. 14 (2010), 2497-2511.

[17] F. Kohsaka, W. Takahashi, Generalized nonexpansive retractions and a proximal-type algorithm in Banach spaces, J. Nonlinear Convex Anal. 8 (2007), 197-209.

[18] F. Kohsaka, W. Takahashi, Fixed point theorems for a class of nonlinear mappings related to maximal monotone operators in Banach spaces, Archiv der Mathematik 91 (2008), 166-177.

[19] W. Takahashi, Nonlinear Functional Analysis, Yokohama Publishers, Yokohama, 2000.

[20] W. Takahashi, Fixed point theorems for new nonlinear mappings in a Hilbert space, J. Nonlinear Convex Anal. 11 (2010), 79-88.

[21] W. Takahashi, Y. Takeuchi, Nonlinear ergodic theorem without convexity for generalized hybrid mappings in a Hilbert space, J. Nonlinear Convex Anal. 12 (2011), 399-406.

[22] W. Takahashi, N.-C. Wong, J.-C. Yao, Attractive point and mean convergence theorems for new generalized non- spreading mappings in Banach spaces, Infinite Products of Operators and Their Applications (S. Reich and A. J. Zaslavski, eds.), Contemporary Mathematics, vol. 636, American Mathematical Society, Providence, 2015, pp. 225-248. 